du Sud-Est porteur de la mutation S163R, qui serait peut-être apparue par sélection au traitement par la méfloquine, produit voisin de l'halofantrine, et qui semblerait sensible à la chloroquine. Le rôle de l'amantadine, en revanche, n'a pu être étudié qu'in vitro et s'est avéré variable vis-à-vis de différentes souches. Comme la chloroquine, cette molécule - avec un pK de 9,0 serait expulsée de la vacuole digestive acide et concentrée dans le cytoplasme, se liant à une cible différente de celle de la chloroquine, non identifiée à l'heure actuelle. La mutation compensatrice S163R, en rendant l'efflux plus difficile, expliquerait l'apparition de la résistance. Si les faits présentés sont convaincants, ils laissent cependant des inconnues concernant leur interprétation et T.R. Wellems présente une autre explication (Figure 1) [1]. Le rôle de l'amantadine s'expliquerait par sa fonction d'inhibiteur de canal $\mathrm{H}^{+}$. Sensible aux mutations, elle se lierait à un acide aminé du pore PfCRT d'un parasite résistant à la chloroquine. Le profil exact des mutations a certainement son importance, expliquant pourquoi le produit agit mieux sur une souche que sur une autre. La mutation S163R aurait un double effet: s'opposer à la liaison de l'amantadine et, par une charge positive, empêcher l'efflux de chloroquine et restaurer la sensibilité. Le mode d'action de I'halofantrine est sans doute différent de celui de l'amantadine, mal explicité actuellement. On peut cependant remarquer que si la mutation S163R se retrouve dans l'évolution de l'une et l'autre résistance, elle s'accompagne de mutations différentes dans les deux cas, mutations qui semblent nécessaires à l'efficacité des molécules. Ces haplotypes divers pourraient refléter des modes d'interaction distincts avec PfCRT.

y a-t-il, comme le suggèrent D.J. Johnson et al. [2], une compétition entre la chloroquine et l'amantadine pour un efflux de molécule chargée? S'agit-il d'une toxicité directe de l'amantadine et de l'halofantrine? Les travaux ultérieurs
NOUVELLE

\section{Un nouveau modèle de production des cellules souches hématopoïétiques intra-embryonnaires}

Ana Cumano, Isabelle Godin nous le diront sans doute. Une perspective plus appliquée est celle de la recherche de molécules ciblant PfCRT comme l'amantadine, et pouvant être employées conjointement à la chloroquine pour traiter les sujets infestés tant par des souches résistantes que par des souches sensibles. $\diamond$

Plasmodium chloroquine resistance: towards a specific attack?

\section{RéFÉRENCES}

1. Wellems TE. Transporter of a malaria catastrophe. Nat Med 2004; 10: 1169-71.

2. Johnson DJ, Fidock DA, Mungthin M, et al. Evidence for a central role for PfCRT in conferring Plasmodium falciparum resistance to diverse antimalarial agents. Mol Cell 2004; 15: 867-77.

3. Fidock DA, Nomura T, Tailey AK, et al. Mutations in the $P$. falciparum digestive vacuole transmembrane protein PfCRT and evidence for their role in chloroquine resistance. Mol Cell 2000; 6: 861-71.

4. Tran CV, Saler MH. The principal chloroquine resistance protein of Plasmodium falciparum is a member of the drug/metabolite transporter family. Microbiology 2004; 150: 1-3.

5. Sidhu ABS, Verdier-Pinard D, Fidock DA. Chloroquine resistance in Plasmodium falciparum malaria parasites conferred by pfcrt mutations. Science 2002; 298: 210-3.

\section{A. Cumano: Unité de}

Développement des

lymphocytes, Inserm U.668,

Institut Pasteur,

25 , rue du Docteur Roux,

75724 Paris Cedex 15,

France.

I. Godin : Inserm U.362,

Institut Gustave Roussy,

39 , rue Camille Desmoulins,

94805 Villejuif, France.

cumano@pasteur.fr

production de cellules sanguines. Les précurseurs formés un peu plus tardivement dans le compartiment intra-embryonnaire (dans une région qui comprend l'aorte, les gonades et le mésonéphros, appelée AGM) possèdent, quant à eux, les caractéristiques de multipotentialité et d'auto-renouvellement à long terme qui caractérisent les CSH [1].

Bien que les données cytologiques et fonctionnelles obtenues in vitro et in vivo s'accordent pour désigner l'aorte et 
le mésenchyme sous-jacent comme le lieu d'origine des CSH, la localisation précise de leur site d'émergence n'a pas encore été déterminée. L'analyse cytologique de l'AGM au moment de la production de CSH (pour revue, voir [1]), montre l'existence d'agrégats composés de cellules hématopoïétiques immatures, localisés sur les parois ventrolatérales de l'aorte, et de plages cellulaires localisées sous les îlots aortiques, qui expriment, entre autres, l'antigène AA4.l et le facteur de transcription GATA-3, et qui n'expriment pas le marqueur caractéristique des cellules hématopoïétiques CD45. Alors que les «îlots aortiques » ont été décrits chez les embryons de tous les vertébrés, y compris l'embryon humain, les plages cellulaires ont été caractérisées jusqu'à présent uniquement chez l'embryon de souris [2] et chez I'humain [3].

\section{Comment se forment les CSH intra-embryonnaires?}

La nature des processus conduisant à la production des CSH au niveau de l'aorte fait actuellement l'objet de controverses.

L'ensemble des données cytologiques (pour revue, voir [1]), indique que les précurseurs intra-embryonnaires, comme ceux du sac vitellin, expriment un grand nombre de protéines et de gènes également exprimés par les cellules endothéliales (CD31, CD34, c-kit, AA4.1, GATA-2, entre autres). Par ailleurs, des expériences pratiquées in vivo et in vitro ont montré que les CSH intra-embryonnaires expriment c-kit, CD34, CD31 et AA4.1. Enfin, des données plus récentes indiquent que des cellules qui n'expriment pas CD45 sont capables de produire des cellules sanguines de tous les lignages. L'absence d'expression de CD45, jumelée à l'expression de marqueurs endothéliaux ont conduit à considérer que l'endothélium aortique pouvait donner naissance aux CSH intra-embryonnaires (modèle de « l'endothélium hématogène $\gg)[4-8]$.
Apports d'une étude multiparamétrique : phénotype, fonction et expression

Nous avons caractérisé [9], par cytométrie de flux, dans l'AGM d'embryons de souris, une population de cellules CD45-, c-kit ${ }^{+}, \mathrm{AA} 4 . \mathrm{l}^{+}, \mathrm{CD}_{3} \mathrm{l}^{+}, \mathrm{CD} 4 \mathrm{l}^{+}$. Cette population comprend la quasi-totalité des précurseurs multipotents précédemment quantifiés dans I'AGM. II s'agit bien de $\mathrm{CSH}$, puisque ces cellules sont capables de reconstituer à long terme le système hématopoïétique de souris alymphoïdes, dépourvues de cellules natural killer.

L'analyse des CSH purifiées à partir de I'AGM montre qu'elles expriment des marqueurs de surface (CD31, CD34, ckit, AA4.1) et des facteurs de transcription (Lmo2, GATA-2) communs aux lignages hématopoïétiques et endothéliaux. Mais elles se distinguent des cellules endothéliales par l'expression de CD4l, récemment identifié comme un marqueur des précurseurs hématopoïétiques embryonnaires [10], et du facteur de transcription GATA-3, tous deux absents des cellules endothéliales.

L'ensemble des marqueurs de CSH ainsi définis a été corrélé avec les profils d'expression précédemment obtenus in situ chez l'embryon [2] et complété par une cartographie phénotypique en microscopie confocale [9]. La combinaison de

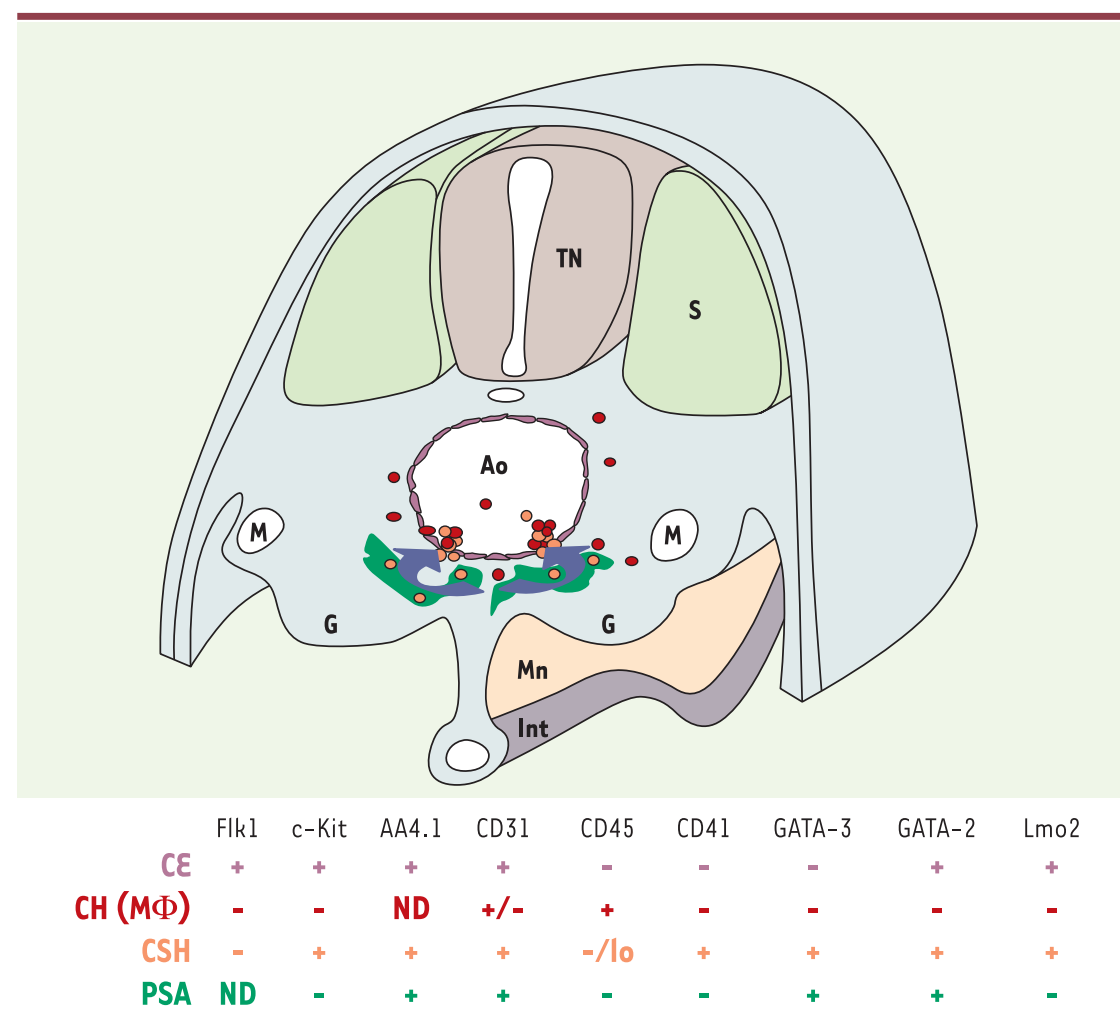

Figure 1. Distribution des gènes et marqueurs membranaires exprimés par les cellules hématopoïétiques et endothéliales dans la région aortique de l'embryon de souris. La combinaison de gènes et marqueurs de surfaces exprimés par les cellules souches hématopoḯtiques (CSH, en orange) purifiées à partir d'une région appelée AGM, qui comprend l'aorte (Ao), les gonades (G) et le mésonéphros $(M)$, révèle exclusivement des cellules faisant partie des îlots aortiques ou des plages sous-aortiques. Les flèches bleues indiquent le sens de migration présomptif des CSH, depuis les plages sous-aortiques (PSA, en vert) vers la lumière de l'aorte où elles contribuent aux îlots, avant d'être libérées dans la circulation et de coloniser le foie fœtal. CE : cellules endo-

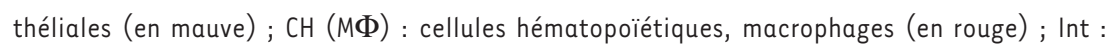
intestin ; Mn : mésentère ; $S$ : somites; TN : tube neural. 
marqueurs qui définissent les CSH est bien localisée au niveau des îlots aortiques, mais également par des cellules localisées au sein des plages sous-aortiques (Figure 1).

\section{Définition d'un modèle alternatif de production des CSH}

L'ensemble des données que nous avons obtenues [9] montrent que les CSH, identifiées par leur phénotype (CD45-, C$\left.\mathrm{kit}^{+}, \mathrm{AA} 4 . \mathrm{I}^{+}, \mathrm{CD} 4 \mathrm{l}^{+}, \mathrm{CD} 3 \mathrm{I}^{+}\right)$sont localisées, non seulement dans le plancher aortique, mais aussi dans le mésenchyme sub-aortique. Ces résultats désignent donc les plages sous-aortiques comme le site de production des CSH, et non pas le plancher aortique, où la présence de CSH semble plutôt refléter leur libération dans la circulation sanguine, préalable à la colonisation du foie fœtal. Les perspectives ouvertes par ce travail [9], et en particulier la caractérisation de la fonction des composants des plages sous-aortiques dans la formation des CSH, pourraient déboucher sur l'identification de signaux impliqués dans la différenciation de cellules mésodermiques en dérivés hématopoiétiques. Ces connaissances seront fondamentales pour la manipulation des cellules souches embryonnaires dans leur différenciation vers la voie hématopoiétique. $\diamond$

A new model for intra-embryonic

hematopoietic stem cell generation

\section{RÉFÉRENCES}

1. Godin I, Cumano A. The hare and the tortoise: an embryonic haematopoietic race. Nat Rev Immunol 2002; 2: 593-604.

2. Manaia A, Lemarchandel V, Klaine M, et al. Lmo2 and GATA- 3 associated expression in intraembryonic hemogenic sites. Development 2000; 127: 643-53.

3. Marshall CJ, Moore RL, Thorogood P, et al. Detailed characterization of the human aorta-gonadmesonephros region reveals morphological polarity resembling a hematopoietic stromal layer. Dev Dyn 1999; 215: 139-47.
4. Jaffredo T, Gautier R, Eichmann A, Dieterlen-Lievre F. Intraaortic hemopoietic cells are derived from endothelial cells during ontogeny. Development 1998 ; 125: 4575-83.

5. Nishikawa SI, Nishikawa S, Kawamoto $H$, et al. In vitro generation of lymphohematopoietic cells from endothelial cells purified from murine embryos. Immunity 1998; 8: 761-9.

6. De Bruijn MF, Ma X, Robin C, et al. Hematopoietic stem cells localize to the endothelial cell layer in the midgestation mouse aorta. Immunity 2002; $16: 673-83$.

7. North TE, de Bruijn MF, Stacy T, et al. Runx1 expression marks long-term repopulating hematopoietic stem cells in the midgestation mouse embryo. Immunity 2002; 16: 661-72.

8. Oberlin $\varepsilon$, Tavian M, Blazsek I, Peault B. Bloodforming potential of vascular endothelium in the human embryo. Development 2002; 129: 4147-57.

9. Bertrand JY, Giroux S, Golub R, et al. Characterization of purified intraembryonic hematopoietic stem cells as a tool to define their site of origin. Proc Natl Acad Sci USA 2005; 102: 134-9.

10. Mikkola HK, Fujiwara Y, Schlaeger TM, et al. Expression of CD41 marks the initiation of definitive hematopoiesis in the mouse embryo. Blood 2003; 101: 508-16.

\section{NOUVELL $\varepsilon$}

\section{Un nouveau partenaire de MuSK à la jonction neuromusculaire, la 14-3-3 $\gamma$, réprime la transcription des gènes synaptiques}

Laure Strochlic, Annie Cartaud, Jean Cartaud

\section{Biologie cellulaire}

des membranes,

Institut Jacques Monod,

CNRS UMR 7592, Universités

Paris 6 et Paris 7,

2, place Jussieu,

75251 Paris Cedex 05, France. cartaud@ijm.jussieu.fr

plexes de signalisation entre le motoneurone et la fibre musculaire sont nécessaires à la coordination de la différenciation pré- et postsynaptique.

La formation de l'appareil postsynaptique requiert, outre le récepteur de l'ACh, au minimum trois

> Les jonctions neuromusculaires s'établissent à la suite d'interactions complexes entre les terminaisons axonales des motoneurones, les fibres musculaires et les cellules de Schwann. La genèse de la jonction neuromusculaire culmine avec la formation de deux domaines spécialisés du nerf et du muscle, la terminaison présynaptique impliquée dans la libération de l'acétylcholine (ACh), et l'appareil postsynaptique responsable de la reconnaissance de l'ACh et de la dépolarisation de la membrane. La membrane postsynaptique assure ces fonctions sur la base d'une accumulation de récepteurs de l'ACh. L'efficacité de la transmission synaptique est renforcée par la juxtaposition précise des sites de libération de I'ACh (les zones actives présynaptiques) et des récepteurs postsynaptiques qui assure leur activation rapide (de l'ordre de la microseconde). Une telle organisation suggère que des mécanismes com- composants : l'agrine, un héparane sulfate protéoglycane sécrété par le nerf, un récepteur tyrosine kinase spécifique du muscle (MuSK), la pièce centrale du complexe récepteur de l'agrine, et la rapsyne, une protéine extrinsèque nécessaire à l'agrégation du récepteur de l'ACh [1]. L'activation de MuSK (par phosphorylation de tyrosines) par l'agrine déclenche en aval une cascade d'événements aboutissant au recrutement du récepteur de l'ACh via la rap- 